\title{
Inter-day reliability of a Wattbike cycle ergometer sprint protocol in male rugby players
}

\author{
Simon Cushman, Richard Bott, Craig Twist, Jamie Highton
}

Objective: The purpose of this study was to establish the inter-day reliability of several performance variables measured during a $2 \times 6 \mathrm{~s}$ sprint on a Wattbike cycle ergometer in male rugby players. Reliablilty was assessed against an 'analytical goal' of detecting meaningful changes that might be expected with fatigue in rugby.

Design \& Methods: Sixteen male rugby players performed three trials of the protocol on different days seperated by a mimimum of $48 \mathrm{~h}$. The protocol comprised $2 \times 6 \mathrm{~s}$ 'all out' cycle sprints with $90 \mathrm{~s}$ recovery between efforts. Typical error (TE), coefficient of variation (CV), smallest worthwhile change (SWC) and moderate change (MC) were calculated between each trial for seven variables.

Results: Peak Power output significantly increased from the first to the third trial (3.3-4.4 \%), whilst all variables had better reliability from trials 2-3 than 1-2. Mean power (3.7\%), peak power (4.2\%) and total distance $(2.7 \%)$ had a CV\% lower than decrements observed with team sport fatigue $(\sim 5 \%)$, however only total work had a TE $(2.1 \mathrm{~kJ})$ lower than the smallest worthwhile change $(4.6 \mathrm{~kJ})$ while the remainder were lower than the moderate worthwhile change.

Conclusion: Assuming one complete habituation trial is completed, total work appears to be the most reliable variable capable of detecting a worthwhile change in performance. We recommend using this variable to monitor fatigue in rugby players.

(Journal of Trainology 2018;7:1-4)

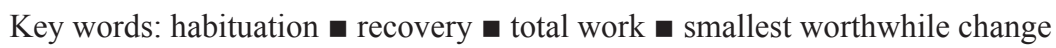

\section{INTRODUCTION}

Rugby players can be subjected to high amounts of external training and match loads, leading to extended periods of fatigue. ${ }^{1,2}$ Force and power production (neuromuscular function) is frequently assessed when determining fatigue and recovery in rugby players, often evidenced as a reduction in maximal vertical jump performance., ${ }^{3,4}$ However, jump performance can remain unchanged after prolonged intermittent activity despite the presence of other indicators of fatigue. ${ }^{5}$ Thus, a single measure of neuromuscular function might not be suffiicient to detect changes in specific components of neuromuscular power production. ${ }^{6}$

Peak power output from cycle ergometer sprint tests has been used to quantify fatigue in team sports. ${ }^{7,8}$ Protocols comprise short bouts ( $3-10 \mathrm{~s})$ of 'all-out' efforts to provide a measure of peak lower limb power that is independent of an imposed body mass load and does not involve eccentric loading of the musculature. Whilst such a measure is attractive to practitioners looking to minimize additional fatigue and risk of injury, the use of peak power alone might not provide a complete picture of the neuromuscular characteristics of an athlete. ${ }^{9}$ Indeed, peak power was unaffected by an intensified period of training in rugby league players ${ }^{10}$ and the force and velocity components of neuromuscular function are affected differently in the days after a rugby league match or controlled muscle damaging protocol. ${ }^{6,11}$ Gathercole et al. ${ }^{9}$ have also argued that typical assessments of neuromuscular function (including peak power) might lack sufficient sensitivity to detect some of the key adaptations associated with fatigue. The evaluation of several variables associated with sprint cycling that enables assessing several neuromuscular characteristics, e.g. peaks and means for cadence and force, as well as capacity measures such as distance and total work, is therefore warranted. This could in turn influence how a subsequent training session is structured to focus more on force, speed or capacity to provide maximum benefit to athletic development and performance.

The Wattbike cycle ergometer provides an easy to use method that is readily available to most rugby clubs and sport science labortories and has become an increasingly popular training and testing tool. Acceptable reliability $(\mathrm{CV}<5 \%)$ of power output during maximal effort cycling in trained cyclists has been reported. ${ }^{7}$ The reliability of peak power measures on a Wattbike using short-term, all out tests in team sport players also possesses acceptable reliability $(\sim 3.0 \%) .{ }^{12}$ However, this study assessed the reliability of only peak power and was performed in team sport players accustomed to sprinting on a Wattbike as part of their regular training. Additionally, the researchers took the absolute peak power value, whereas it has been suggested that reliability of cycle tests can be improved by averaging two bouts. ${ }^{13}$ Understanding the reliability of a range of neuromuscular measures and the extent to which players unaccustomed to a test require habituation would therefore be practically meaningful. Accordingly, this study

Received September 20, 2017; accepted January 25, 2018

From the Department of Sport and Exercise Sciences, University of Chester, United Kingdom (S.C., R.B., C.T., J.H.)

Communicated by Takashi Abe, Ph.D.

Correspondence to Dr. Craig Twist, University of Chester, Parkgate Road, Chester, CH1 4BJ, England. Email: c.twist@chester.ac.uk

Journal of Trainology 2018;7:1-4 @2012 The Active Aging Research Center http://trainology.org/ 
sought to establish the reliability of key performance measures during a $2 \times 6 \mathrm{~s}$ 'all-out' Wattbike test in male rugby players.

\section{MATERIALS AND METHODS Experimental subjects}

Sixteen competitive male rugby players volunteered for this study (age $23.7 \pm 4.8$ years, stature $180 \pm 6.3 \mathrm{~cm}$, body mass $88.6 \pm 14.0 \mathrm{~kg}$ ). Each participant provided written informed consent. The study received ethics approval from the Faculty of Life Sciences ethic committee at the University of Chester.

\section{Experimental approach to the problem}

Participants attended the laboratory on three separate occasions at a similar time of day $( \pm 1 \mathrm{~h})$, with each visit separated by $6 \pm 2$ days. Each visit consisted of a 5 min warm-up followed by $2 \times 6 \mathrm{~s}$ 'all out' cycle sprints then a cool down. Participants were instructed to refrain from caffeine $12 \mathrm{~h}$ hour before tests and to attend each visit to the laboratory in a wellhydrated state. Participants were asked to consume a normal diet and refrain from supplementation $24 \mathrm{~h}$ before each trial.

All cycle tests were completed on the same air-braked cycle ergometer (Wattbike Ltd, Nottingham, UK) sampling at 100 $\mathrm{Hz}$. Before each test the ergometer was calibrated according to the manufacturer's instructions. Measurements of ergometer set up were recorded for each participant to ensure saddle and handlebar height remained constant for all visits.

\section{Procedures}

Each visit consisted of a standardized 5 min warm up cycling at $100-150 \mathrm{~W}$ with a $3 \mathrm{~s}$ all out sprint at $3 \mathrm{~min}$, with cadence and resistance freely chosen throughout. After a passive rest period $(\sim 5 \mathrm{~min})$, the ergometer was set at a fixed resistance of level 2 on the magnetic brake and level 6 on the air brake based on pilot work. Participants then performed a $6 \mathrm{~s}$ all-out sprint from a stationary start. For each trial the test started with the pedals in a horizontal position and the dominant foot in the forward position. This was immediately followed by a $90 \mathrm{~s}$ active recovery period of easy pedaling at a self-selected resistance $(\mathrm{rpm}<80)$. The participant then repeated the $6 \mathrm{~s}$ sprint followed by a $5 \mathrm{~min}$ cool down period. Verbal encouragement to provide maximum effort was given by the researcher throughout each trial. Measures of peak power (the highest power output from the two sprints, W), mean peak power (the mean of the peak power achieved in the two sprints of a trial), mean power (the mean power achieved throughout the two sprints of a trial), peak force $(\mathrm{N})$, peak cadence $(\mathrm{rpm})$, total work done $(\mathrm{kJ})$, and total distance covered (m) were recorded.

\section{Statistical analysis}

Reliability of each measure was assessed using the typical error (TE) and coefficient of variation (CV\%), both of which were expressed with $90 \%$ confidence limits (in parantheses). The TE was calculated as the standard deviation (SD) of the differences between each trial divided by $\sqrt{ } 2$ and the $\mathrm{CV} \%$ as; $(\mathrm{TE} /$ grand mean $) \times 100$. The smallest worthwhile change (SWC) in performance was considered as $0.2 \times$ the shared standard deviation of scores for a given variable. ${ }^{14}$ Moderate changes (MC) were subsequently calculated as $3 \times \mathrm{SWC}^{15}$ To evaluate any apparent systematic differences, ANOVA were performed on the test re-test data with significance set at 0.05 for each statistical process. In the presence of a statistically significant $F$ ratio, post-hoc paired samples $t$-tests were performed to establish where differences were. All data were analysed using SPSS (IBM, SPSS, version 22).

\section{RESULTS}

Reliability of performance variables across the three trial days is presented in Table 1 . Of all variables analysed, only total work had a TE $(\sim 1.6-2.9 \mathrm{~kJ})$ lower than the SWC $(\sim 4.6$ $\mathrm{kJ})$. However, all other variables possessed a TE lower than the calculated moderate change in performance. The $\mathrm{CV} \%$ was lowest for distance covered $(\sim 2.7 \%)$ and highest for peak cadence $(\sim 6.0 \%)$.

Between day comparisons indicated that peak power and mean power increased significantly up to trial 3, whereas mean peak power increased significantly at trial 2 with no further improvement. No other variables systematically changed across days. Specific comparisons of variability between days indicated that reliability was, for the most part, best when comparing days 2 and 3 (Table 2). Again, only variability of total work between different days was lower than the SWC.

Table 1 Inter-day reliability of performance variables from repeated sprints on the Wattbike cycle ergometer. Values are mean $\pm \mathrm{s}$ with $90 \%$ confidence limits in parentheses.

\begin{tabular}{lccccccc}
\hline & Trial 1 & Trial 2 & Trial 3 & TE & SWC & MC & CV\% \\
\hline Peak power (W) & $1378.6 \pm 226.4$ & $1390 \pm 201.9$ & $1439.8 \pm 196.9^{*} \dagger$ & $53.5(41.9-75.7)$ & 41.8 & 125.3 & $4.2(3.3-6.0)$ \\
Mean peak power (W) & $1208.4 \pm 208.2$ & $1249.9 \pm 164.5^{*}$ & $1289.0 \pm 154.7^{*}$ & $56.1(44.0-79.3)$ & 35.5 & 106.4 & $5.1(4.0-7.3)$ \\
Mean power (W) & $1344.8 \pm 214.9$ & $1363.8 \pm 192.5^{*}$ & $1409.9 \pm 202.5^{* \dagger} \dagger$ & $46.7(36.6-66.1)$ & 40.7 & 122.1 & $3.7(2.9-5.3)$ \\
Peak force (N) & $983.9 \pm 152.9$ & $1012.8 \pm 13.7$ & $1016.9 \pm 134.3$ & $47.9(37.5-67.7)$ & 28.1 & 84.4 & $5.0(3.9-7.2)$ \\
Peak cadence (rpm) & $149.2 \pm 20.6$ & $145.8 \pm 5.9$ & $150.1 \pm 9.3$ & $9.8(7.7-13.9)$ & 2.7 & 8.1 & $6.0(4.7-8.6)$ \\
Total distance (m) & $222.5 \pm 17.2$ & $222.5 \pm 17.7$ & $224.4 \pm 17.9$ & $5.8(4.6-8.3)$ & 3.5 & 10.6 & $2.7(2.1-3.9)$ \\
Total work (kJ) & $47.2 \pm 22.7$ & $48.0 \pm 22.9$ & $49.0 \pm 23.1$ & $2.1(1.6-2.9)$ & 4.6 & 13.7 & $4.8(3.7-6.8)$ \\
\hline
\end{tabular}

$T E=$ typical error. $C V \%=$ coefficient of variation. $S W C=$ smallest worthwhile change, calculated as $0.2 \times$ pooled standard deviation of scores for that variable . $M C=$ moderate change, calculated as the SWC $\times 0.3$. * Significantly $(P<0.05)$ different to trial 1 , † significantly different to trial 2. 
Table 2 Inter-day comparisons of performance variables. Values are mean $\pm s$ with $90 \%$ confidence limits in parentheses.

\begin{tabular}{|c|c|c|c|c|c|c|c|c|}
\hline & \multicolumn{2}{|c|}{ Trial 1-2 } & \multicolumn{2}{|c|}{ Trial 1-3 } & \multicolumn{2}{|c|}{ Trial 2-3 } & \multirow{2}{*}{ SWC } & \multirow{2}{*}{ MC } \\
\hline & TE & CV\% & TE & $\mathrm{CV} \%$ & TE & CV\% & & \\
\hline Peak power $(W)$ & $57.7(42.6-89.3)$ & $4.7(3.5-7.4)$ & $71.5(52.9-110.7)$ & $5.9(4.3-9.3)$ & $48.9(36.1-75.7)$ & $3.7(2.7-5.8)$ & 41.8 & 125.3 \\
\hline Mean peak power $(\mathrm{W})$ & $51.0(37.7-78.9)$ & $5.2(3.8-8.1)$ & $84.1(62.1-130.1)$ & $7.9(5.7-12.4)$ & $60.8(44.9-94.0)$ & $5.0(3.7-7.8)$ & 35.5 & 106.4 \\
\hline Mean power (W) & $48.8(36.0-75.5)$ & $4.1(3.0-6.4)$ & $68.4(50.5-105.9)$ & $5.7(4.2-8.9)$ & $44.6(32.9-69.0)$ & $3.2(2.4-5.1)$ & 40.7 & 122.1 \\
\hline Peak force (N) & $55.0(40.7-85.2)$ & $6.1(4.5-9.6)$ & $58.0(42.8-89.7)$ & $6.0(4.4-9.5)$ & $39.4(29.1-61.0)$ & $3.6(2.7-5.7)$ & 28.1 & 84.4 \\
\hline Peak cadence (rpm) & $12.9(9.5-19.9)$ & $7.9(5.8-12.4)$ & $1.7(1.3-2.6)$ & $8.5(6.2-13.5)$ & $5.2(3.6-8.1)$ & $3.3(2.5-5.2)$ & 2.7 & 8.1 \\
\hline Total distance (m) & $5.6(4.2-8.7)$ & $2.7(2.0-4.2)$ & $5.4(4.0-8.3)$ & $2.5(1.8-3.9)$ & $6.1(4.8-9.4)$ & $2.7(2.0-4.3)$ & 3.5 & 10.6 \\
\hline Total work (kJ) & $1.2(0.9-1.9)$ & $4.5(3.3-7.1)$ & $2.7(2.0-4.2)$ & $6.0(4.4-9.4)$ & $2.6(1.9-4.1)$ & $5.0(3.7-7.9)$ & 4.6 & 13.7 \\
\hline
\end{tabular}

$T E=$ typical error $C V \%=$ coefficient of variation. $S W C=$ smallest worthwhile change, calculated as $0.2 \times$ pooled standard deviation of scores for that variable . $M C=$ moderate change, calculated as the $S W C \times 0.3$.

\section{DISCUSSION}

Peak, mean peak and mean power output systematically increased from the first to the last trial by $4.4 \%, 1.4 \%$ and $3.3 \%$, respectively. Furthermore, in all but one variable (total work), agreement between measurements, according to the $\mathrm{CV} \%$, improved when comparing trials 2-3 compared to $1-2$, indicating that performance was more stable after the first trial. Notably, measurements of peak power continued to increase from trials 2-3, perhaps indicating that team sport athletes who are not accustomed to cycle ergometer sprinting require two habituation sessions to achieve consistent performance in this variable. Furthermore, it was only when comparing trials 2-3 in the present study that the reliability of peak power was similar to that reported in the same protocol by Wehbe et al. ${ }^{12}$ in athletes already accustomed to maximal sprints on a cycle ergometer (CV\% 3.7 cf. 3.1\%). Taken together, our data indicate that team sport athletes should be fully habituated to a maximal repeated sprint test on at least one occasion before data collection. This is in agreement with Driller et al. ${ }^{7}$ who reported similar variability in peak and mean power across multiple trials of maximal sprint cycle tests in trained cyclists after one habituation session.

When considering the reliability of performance against an analytical goal, it was only the measurement of total work that had a typical error lower than the smallest worthwhile change in performance $(2.1 \mathrm{cf} .4 .6 \mathrm{~kJ})$. The measurement of total work is calculated from the distance per revolution multiplied by the force, which has presented itself in a cumulative effect, potentially reducing the error of this variable which was suggested to improve reliability. ${ }^{13}$ As such, this variable is likely to be adequately sensitive to detect small meaningful changes in performance. However, all other variables had a typical error that was smaller than an estimated moderate change in performance, and thus practitioners are likely to be able to detect such a change with sufficient confidence that it is not due to biological, mechanical or procedural variation.

Another appropriate analytical goal, given the aim of this study, is to compare the variability of measurements with the typical change associated with post-match fatigue in team sport players. Previous investigations have shown that maximal voluntary contraction-derived peak torque as well as peak torque at two different speeds are reduced by $\sim 10 \%,{ }^{11,16}$ with each showing different time courses to baseline recovery. Vertical jump performance is also reduced by $5-20 \%{ }^{6,16}$ and cycle ergometer-derived peak power is reduced by $\sim 5 \%$. Combined, these studies suggest that reliability, as a best-case scenario, should be $<5 \%$ to confidently detect changes associated with fatigue after rugby match play. When compared across all three trials, this was the case for all variables with the exception of RPM and peak force. However, when trials 2-3 were compared, all variables had a CV\% equal to or smaller than this goal. Thus, we propose that measurements of power, force, cadence, distance and work are sufficiently reliable to detect changes in performance associated with postrugby match fatigue providing participants have been habituated on one occasion. With this in mind the addition of the further variables may enhance our understanding of the mechanisms of recovery and neuromuscular fatigue and provide valuable information on human muscle performance to inform subsequent training sessions and their content. However, further research may need to look at the sensitivity of each of these variables after exercise and compare them to current known measurements.

From a research perspective, the present analysis indicates that a sample size of $\sim 15-20$ participants would be required to detect a $5 \%$ change in each variable. ${ }^{17}$ This sample size is likely to be achievable for most researchers, and is within the range previously used to investigate fatigue in team sport play$\mathrm{ers}^{3,6}$.

Our findings suggest that the previous study evaluating only peak power of the two Wattbike sprints ${ }^{8}$ overlooks the range of neuromuscular measures available during two short 'all-out' efforts. Not only this, but in $\sim 70 \%$ of our trials, the peak power was recorded from the second sprint. Focusing on peak power alone is likely to underestimate the full extent of an individual's neuromuscular capabilities. Indeed, the high levels of reliability shown for total work done and mean power suggest that using combined data from two sprints as previously recommended $^{13}$ provides a more comprehensive insight of an individual's lower limb neuromuscular function. 


\section{CONCLUSION AND PRACTICAL APPLICATION}

The present study has shown that a $2 \times 6 \mathrm{~s}$ sprint cycling test produces several variables that have sufficient reliability to detect changes in neuromuscular function associated with team-sport fatigue and recovery. However, we would specifically encourage the use of one complete habituation trial before data collection, and recommend the use of total work as the most reliable variable capable of detecting the smallest worthwhile change in performance. Future studies might wish to investigate how this and other variables reported in this study are affected by post-match fatigue in team sport athletes.

\section{REFERENCES}

1. Bradley WJ, Cavanagh BP, Douglas W, Donovan TF, Morton JP, Close GL. Quantification of training load, energy intake and physiological adaptations during a rugby pre season: A case study from an elite European rugby union squad. J Strength Cond Res 2015;29:534-544.

2. Weaving D, Marshall P, Earle K, Nevill A, Abt G. Combining internal and external training load measures in professional rugby league. Int J Sports Physiol Perform 2014;9:905-912.

3. Twist C, Waldron M, Highton J, Burt D, Daniels M. Neuromuscular, biochemical and perceptual post-match fatigue in professional rugby league forwards and backs. J Sports Sci 2012;30:359-367.

4. West DJ, Finn CV, Cunningham DJ, Shearer DA, Jones MR, Harrington $\mathrm{BJ}$, et al. The neuromuscular function, hormonal, and mood responses to a professional rugby union match. J Strength Cond Res 2014;28:194-200.

5. Thorlund JB, Aagaard P, Madsen K. Rapid muscle force capacity changes after soccer match play. Int J Sports Med 2009;30:273-278.

6. McLellan CP, Lovell DI, Gass GC. Markers of post-match fatigue in professional rugby league players. J Strength Cond Res 2011;25:10301039.

7. Driller MW, Argus CK, Shing CM. The reliability of a 30 -s sprint test on the Wattbike cycle ergometer. Int J Sports Physiol Perform 2013;8:379383.

8. Wehbe G, Gabbett T, Dwyer D, McLellan C, Coad S. Neuromuscular fatigue monitoring in team sport athletes using a cycle ergometer test. Int $J$ Sports Physiol Perform 2015;10:292-297.

9. Gathercole R, Sporer B, Stellingwerff T, Sleivert G. Alternative countermovement-jump analysis to quantify acute neuromuscular fatigue. Int J Sports Physiol Perform 2015;10:84-92.

10. Coutts AJ, Reaburn P, Piva TJ, Rowsell GJ. Monitoring for overreaching in rugby league players. Eur J Appl Physiol 2007;99:313-324.

11. Twist C, Eston R. The effect of muscle-damaging exercise on maximal intensity cycling and drop jump performance. J Exerc Sci Fitness 2007;5: 79-87.

12. Wehbe G, Gabbett T, Hartwig TB, McLellan CP. Reliability of a cycle ergometer peak power test in running-based team sport athletes: a technical report. J Strength Cond Res 2015; 29: 2050-2055.

13. Watt KK, Hopkins WG, Snow RJ. Reliability of performance in repeated sprint cycling tests. J Sci Med Sport 2002;5:354-361.

14. Batterham AM, Atkinson G. How big does my sample need to be? A primer on the murky world of sample size estimation. Phys Ther Sport 2005;6:153-163.

15. Hopkins WG, Marshall SW, Batterham AM, Hanin J. Progressive statistics for studies in sports medicine and exercise science. Med Sci Sports Exerc 2006;41:3-13.

16. Duffield R, Murphy, A Snape A, Minett GM, Skein M. Post-match changes in neuromuscular function and the relationship to match demands in amateur rugby league matches. J Sci Med Sport 2012;15:238-243.

17. Batterham AM, Hopkins WG. Making meaningful inferences about magnitudes. Int J Sports Physiol Perform 2006;1:50-57. 